in patients with PD, and investigated whether patients are aware of possible adverse effects.

In total, 120 eligible patients from an academic movement disorders center completed a questionnaire regarding nutritional supplement use. Nutritional supplements were defined as any supplement (e.g. vitamin or herb) used in addition to prescription medication.

At the time of data collection, nutritional supplements were being taken by 76 patients (63\%), of whom 46 used $\geq 2$ supplements. Of the patients taking supplements, 99\% were also taking prescription medications. Vitamins and multivitamins were the most common categories of supplements used. Among patients taking supplements, $26 \%$ used them specifically for PD symptoms. Nutritional supplement use could not be predicted by factors such as age, gender, marital status, education level, income and duration of PD. Of patients taking nutritional supplements, only three patients (4\%) were aware of possible adverse effects or drug interactions with prescription medications, and only $47 \%$ consulted their doctor before or after supplement use.

The authors state that health-care providers should ask all PD patients about supplement use, so that potentially harmful drug interactions can be avoided. Further trials are needed to test the effects of supplements in PD patients and in the general population.

Original article Wolfrath SC et al. (2006) Use of nutritional supplements in Parkinson's disease patients. Mov Disord [doi: 10.1002/mds.20902]

\section{Different gene expression profiles for heroin and cocaine abusers}

Drug abuse and addiction result in long-term alterations in the brain's cellular composition, but many of the molecular mechanisms behind these changes are unclear. A recent study conducted in cocaine abusers found a marked downregulation of several myelin-related genes in the nucleus accumbens, and a new study by the same group has investigated whether similar gene expression changes occur in heroin abusers.

Albertson and colleagues investigated the postmortem nucleus accumbens of seven heroin abusers and seven matched controls using high-throughput microarray technology.
All subjects had tested negative for other common drugs of abuse, including cocaine. Analysis of gene expression data was carried out both independently and in relation to the previous data for cocaine abusers $(n=10)$.

Compared with controls, the nucleus accumbens of heroin abusers demonstrated a marked decrease in the number of transcripts encoding proteins involved in presynaptic processes such as vesicle storage and release-a finding that was not mirrored in cocaine abusers. Heroin abusers, however, displayed no downregulation of the genes previously reported in the cocaine cohort, and there was little overlap between the altered gene expression profiles of the two groups.

The authors conclude that although cocaine and heroin abuse both cause an increase in extracellular dopamine levels within the nucleus accumbens, the underlying molecular changes are largely unique to each drug. They suggest that the current emphasis placed on common mechanisms behind the effects of addictive drugs merits re-examination, and that a better understanding of the molecular correlates of addiction might enable the development of novel treatment strategies.

Original article Albertson DN et al. (2006) Distinctive profiles of gene expression in the human nucleus accumbens associated with cocaine and heroin abuse. Neuropsychopharmacology [doi: 10.1038/sj.npp.1301089]

\section{Haloperidol: a rescue medication in the treatment of acute migraine}

Patients with migraine are often given neuroleptics-dopamine-receptor-blocking agentsto combat nausea. Neuroleptics also seem to be more effective than triptans at relieving migraine pain, but are associated with adverse effects. Honkaniemi and co-workers investigated the efficacy and tolerability of the neuroleptic haloperidol, which works mainly by blocking the D2 dopamine receptor, in the treatment of acute or prolonged migraine.

In this randomized, placebo-controlled study, over $80 \%$ of the patients administered intravenous haloperidol received notable relief in pain intensity from the drug, within hours of treatment. Many of these individuals had received previous migraine treatment (nonsteroidal anti-inflammatory drug-type 